chimerism, T-cell chimerism and transplant outcomes was also investigated in those patients with lineage-specific analysis.

Results In total, 186 patients exhibited CC while 98 patients exhibited MC. Mean age at transplant and conditioning were identified as significant predictors of MC. Year of transplant and chimerism status were identified as significant predictors of graft failure. In 29 out of 49 patients with high-level MC, graft failure was developed. Early myeloid complete donor chimerism was identified as a useful predictor of long- term engraftment and a rising donor T-cell chimerism. Subsequently, an algorithm was created for the clinical management of chimerism in non-malignant disease.

Conclusions High-level MC is a better predictor of graft failure than low-level MC. Myeloid chimerism can be used as a reliable indicator of the transplant outcome. T-cell chimerism monitoring can be important in patients with T-cell immunodeficiencies. The algorithm suggested is used to inform and predict graft outcomes as well as the need for specific interventions in patients with a non-malignant disease, whilst utilising lineage- specific (myeloid and T-cell) chimerism.

\section{G364(P) DELAYS TO DIAGNOSIS OF CHILDHOOD CANCER: A QUALITATIVE STUDY OF SPECIALIST HEALTH CARE PROFESSIONALS' VIEWS}

${ }^{1} \mathrm{H}$ Bowyer, ${ }^{2} \mathrm{M}$ Girling, ${ }^{3} \mathrm{C}$ Exley, ${ }^{1} \mathrm{D}$ Tweddle. ${ }^{1}$ Northern Institute of Cancer Research, Newcastle University, Newcastle Upon Tyne, UK; ${ }^{2}$ Institute of Health and Society, Newcastle University, Newcastle Upon Tyne, UK; ${ }^{3}$ Research and Innovation Department, Northumbria University, Newcastle Upon Tyne, UK

\subsection{6/archdischild-2018-rcpch.354}

Aims Specialist health care professionals (SHCPs) working in Paediatric Oncology Principle Treatment Centres (PCTs) can provide invaluable insights into diagnostic pathways and the consequences of delays in diagnosis of childhood cancer. This study is the first to explore their views on the significance of delays in diagnosis of childhood cancer and reports their suggestions of how delays could be reduced.

Methods Semi-structured interviews were conducted with 20 SHCPs working in a variety of paediatric oncology specialist roles within PCTs throughout the UK. SHCPs included clinicians, nurses and allied healthcare professionals. Interviews were audio-recorded, transcribed verbatim and analysed using thematic analysis.

Results SHCPs believe that delays in diagnosis childhood cancer can have important psychological and physical consequences. They believe there are many barriers to an efficient childhood cancer diagnosis within the UK, but especially within the primary-care setting. They recognise that the rarity of childhood cancer and the variability of presenting symptoms may make raising awareness a challenge. SHCPs instead would like to see changes to how community paediatric healthcare is delivered, suggesting the UK re-consider the introduction of primary-care paediatricians and routine surveillance of children.

Conclusion SHCPs believe that considerable changes to how paediatric healthcare is delivered in the UK could improve overall childhood health, but could also reduce delays in diagnosis of many conditions including cancer.

Acknowledgement This study was fully funded by Cancer Research UK and was supported by Children's Cancer and Leukaemia Group (CCLG).

\section{G365(P) RETROSPECTIVE COHORT STUDY OF (P)IOR REVERSIBLE ENCEPHALOPATHY SYNDROME (PRES) IN PAEDIATRIC ONCOLOGY PATIENTS OVER A 10 YEAR PERIOD}

'VMY Wong-Spracklen, 2B Messahel, 'D Krishnakumar. 'Paediatric Neurology, Addenbrooke's Hospital, Cambridge University Hospitals NHS Trust, Cambridge, UK; ${ }^{2}$ Paediatric Oncology and Haematology, Addenbrooke's Hospital, Cambridge, UK

\subsection{6/archdischild-2018-rcpch.355}

Aims Literature regarding (P)ior reversible encephalopathy syndrome (PRES) in paediatric oncology is limited. Clinical symptoms of PRES include seizures, headaches, altered consciousness, and visual disturbances. We describe the seizures in PRES, outcomes and need for anti-epileptic medication of this clinical syndrome.

Methods A retrospective cohort study using data from the East Anglia paediatric oncology database for suspected adverse events (SAE) from chemotherapy was conducted. Oncology patients aged 16 and below, from 1 January 2007 until 15 June 2017 who had developed seizures while undergoing chemotherapy, were included. We identified the chemotherapy administered before onset of seizures, magnetic resonance imaging (MRI) reports, and patient outcomes based on antiepileptic drugs (AED) requirement at 6, 12, 24 months or more after seizure occurrence.

Results 38/420 (9.0\%) paediatric patients undergoing chemotherapy developed seizures. $14 / 38$ (36.8\%) were confirmed to have had PRES. The mean age for PRES-associated seizures was 10.1 years ( \pm SD 3.7 years). The mean interval between administration of chemotherapy and onset of seizures in PRES was 7 days \pm SD 5 days (Median 5 days, range 1-17 days). Chemotherapy agents associated with seizure occurrence in PRES were methotrexate in $10 / 14$ (71.4\%), ifosfamide in $3 / 14$ (21.4\%) and vincristine 1/14 (7.1\%). High signal abnormalities seen on MRI changes due to PRES was seen in occipital-parietal lobe (43\%), frontal lobe (43\%), centrum semiovale (14\%), caudate nucleus (14\%), deep white matter (14\%) and temporal lobe $(7 \%)$. Only $1 / 14(7 \%)$ of PRES patients remained on AED for 11 months, compared to $3 / 24$ (12\%) of non-PRES patients who remained on AED at 5 years follow-up.

Conclusion PRES is an important differential to consider in paediatric oncology population developing seizures. The chemotherapeutic agents associated with PRES are often Methotrexate, Vincristine and Ifosfamide. The outcome for seizures associated with PRES is good as only $7 \%$ require anti-epileptic medication for the first 11 months post-PRES. Further studies should be carried out in identifying prognostic markers, e.g. blood pressure, which may aid early identification of patients at risk of PRES and who may benefit from proactive preventative measures.

\section{G366(P) SYSTEMATIC REVIEW ON THE USE OF HYDROXYCARBAMIDE IN REDUCING TRANSCRANIAL DOPPLER VELOCITIES IN CHILDREN WITH SICKLE CELL ANAEMIA AND WHETHER THIS RESULTS IN STROKE PREVENTION}

S Mason. Clinical School, University of Cambridge, Cambridge, UK

\subsection{6/archdischild-2018-rcpch.356}

Introduction Sickle cell anaemia is a common inherited haemoglobinopathy in which distorted red blood cells cause vasoocclusion and ischaemic-reperfusion injury in various organs. 